\title{
Electronic Departure Approval Requests in ATD-2 Daily Operations
}

Todd J. Callantine

San Jose State University
Robert Staudenmeier

Cavan Solutions, Inc.
Lindsay Stevens

NASA Ames Research Center
Jeremy Coupe

NASA Ames Research Center
Andrew Churchill

Mosaic ATM, Inc. 


\section{Departure Approval Requests}

\section{APREQ}

- Traffic management initiative for applying tactical departure delay

- Traditional method: 'Call-For-Release'

Compliance Window:

\section{Two minutes earlier to one minute later}




\section{Stream Insertion}
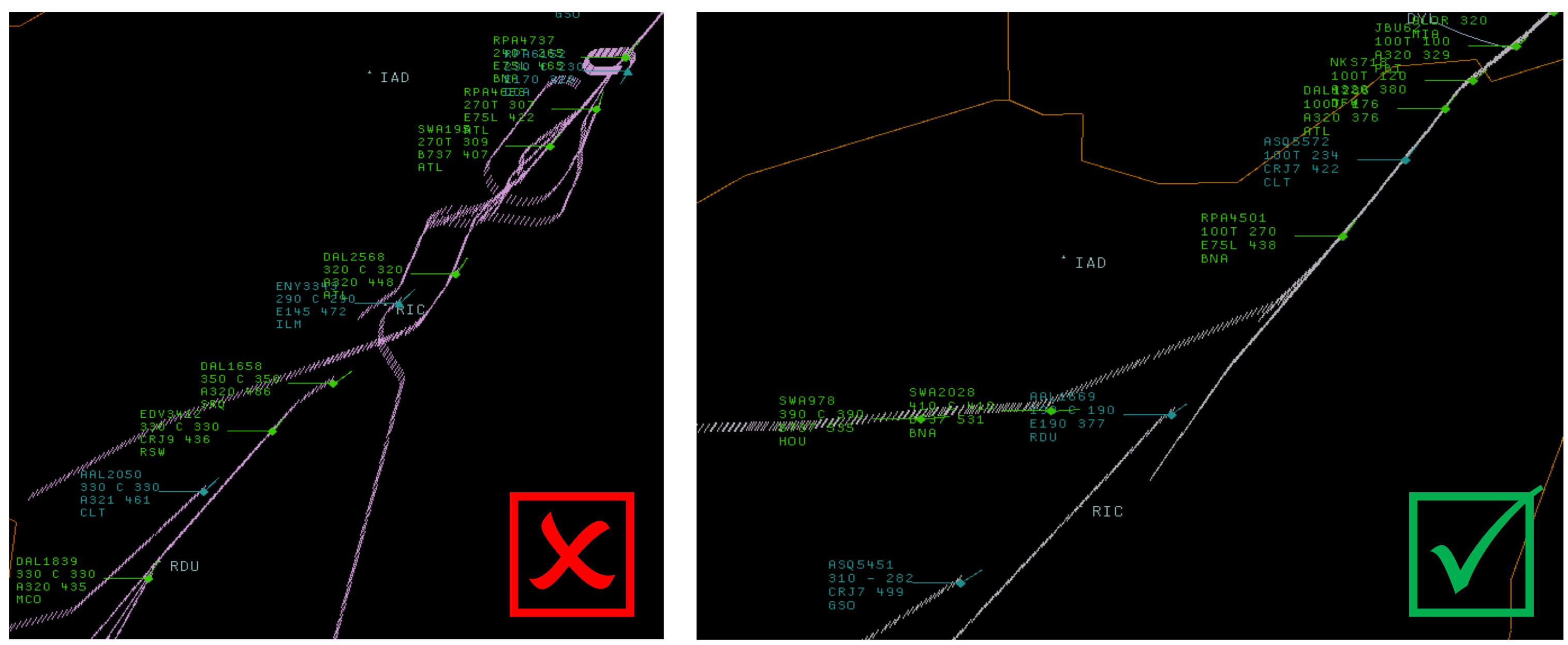


\section{Outline}

- NASA Airspace Technology Demonstration 2 (ATD-2)

- Electronic APREQ negotiation

- Charlotte APREQ flights Jan 2018-Feb 2019 


\section{NASA ATD-2}

Integrated Arrival-Departure-Surface Air Traffic Management

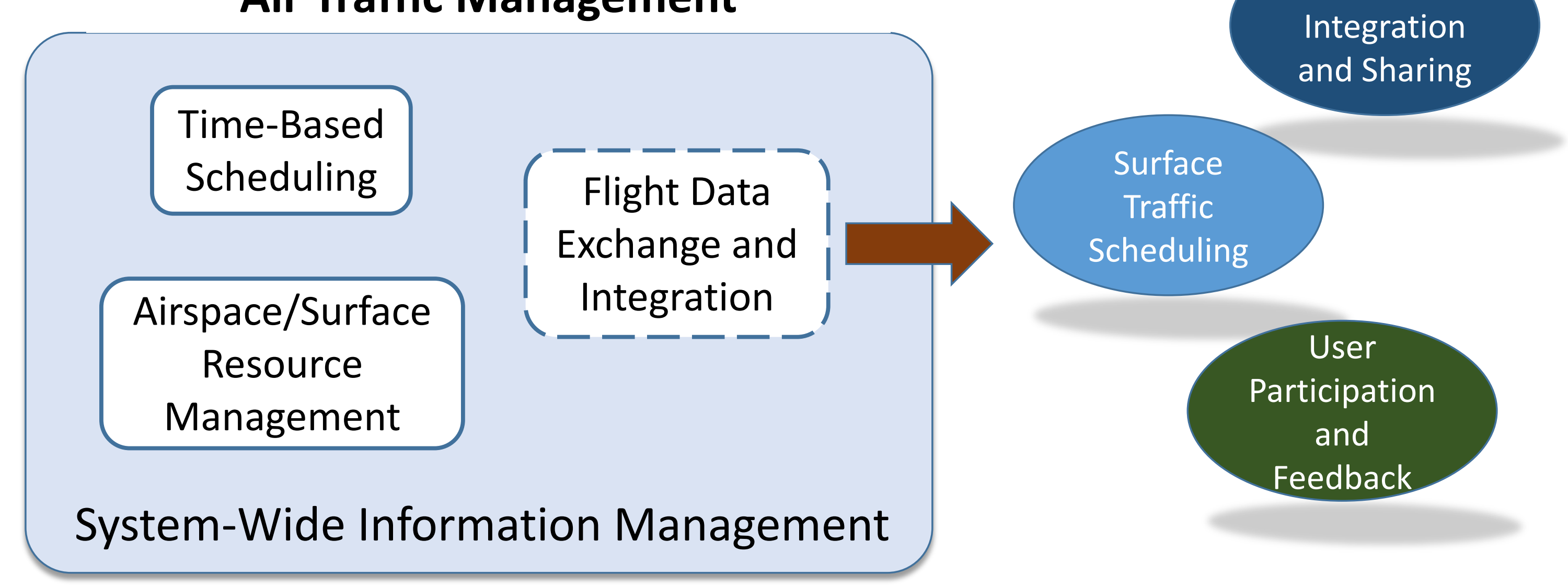




\section{Electronic APREQ Negotiation}

- APREQ flights \& eligibility for electronic negotiation

'STBO Client' Timeline

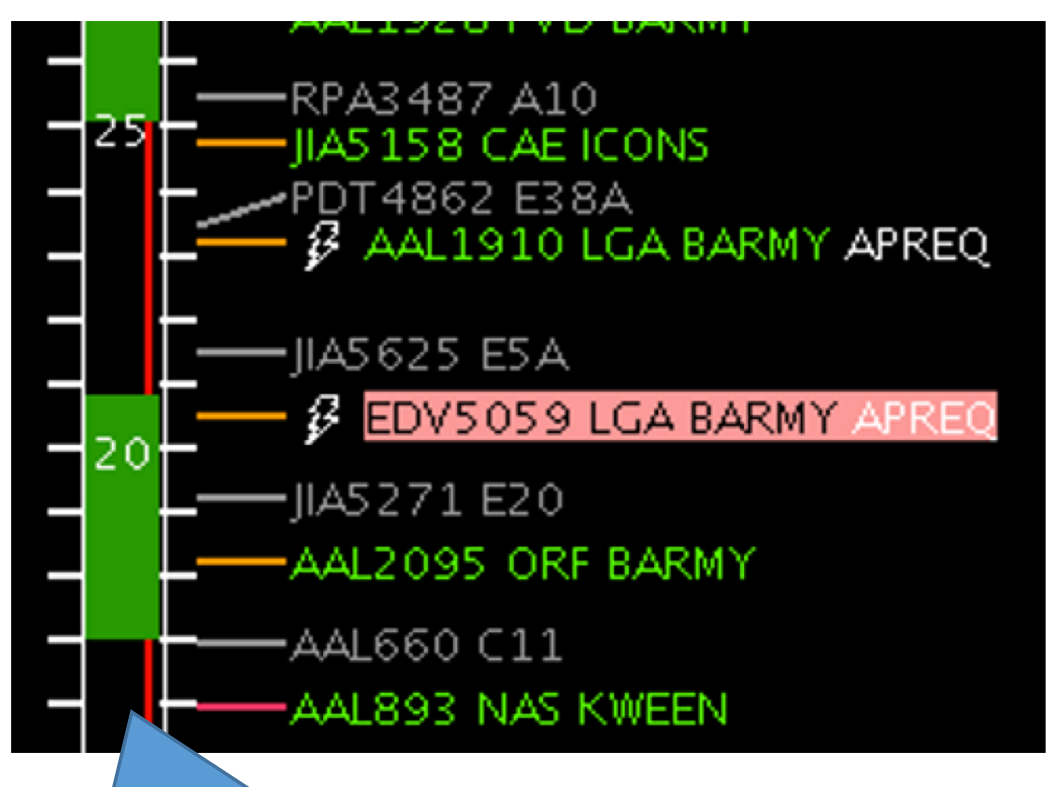

Slot-Availability

Indications

- Semi-automatic or automatic response mode 


\section{Charlotte Operations Jan 2018-Feb 2019}

- 303,729 departures over 402 days (21 low-departure days removed)
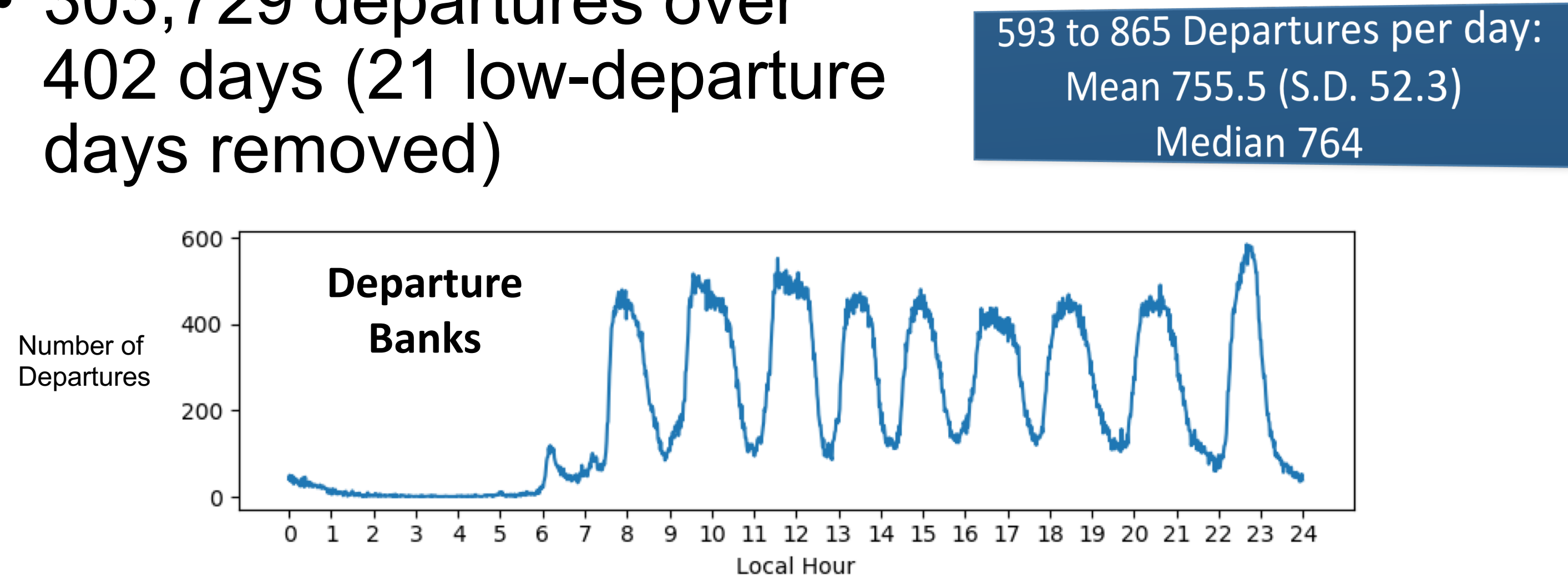

- Top-10 destinations frequently subject to APREQs 


\section{Controlled Release Times}

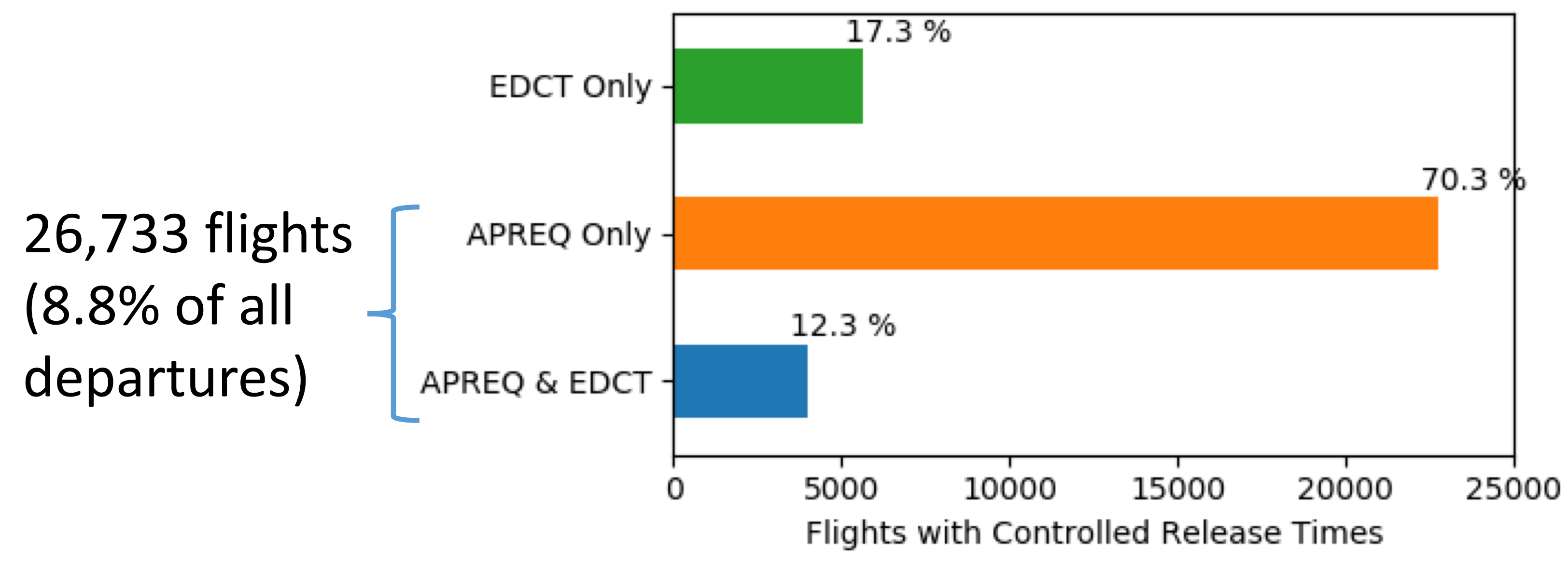




\section{Release Requests: Washington Center}

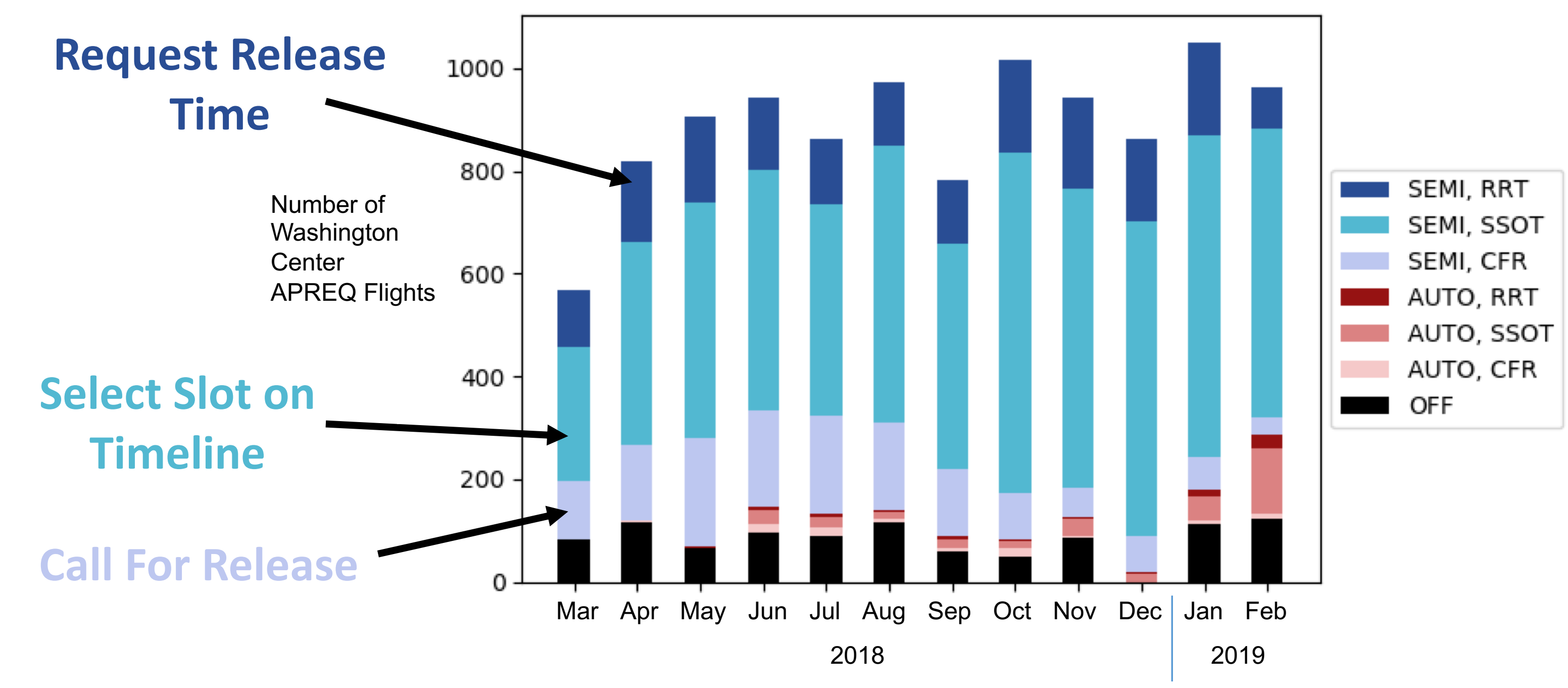




\section{Release Requests: Atlanta Center}

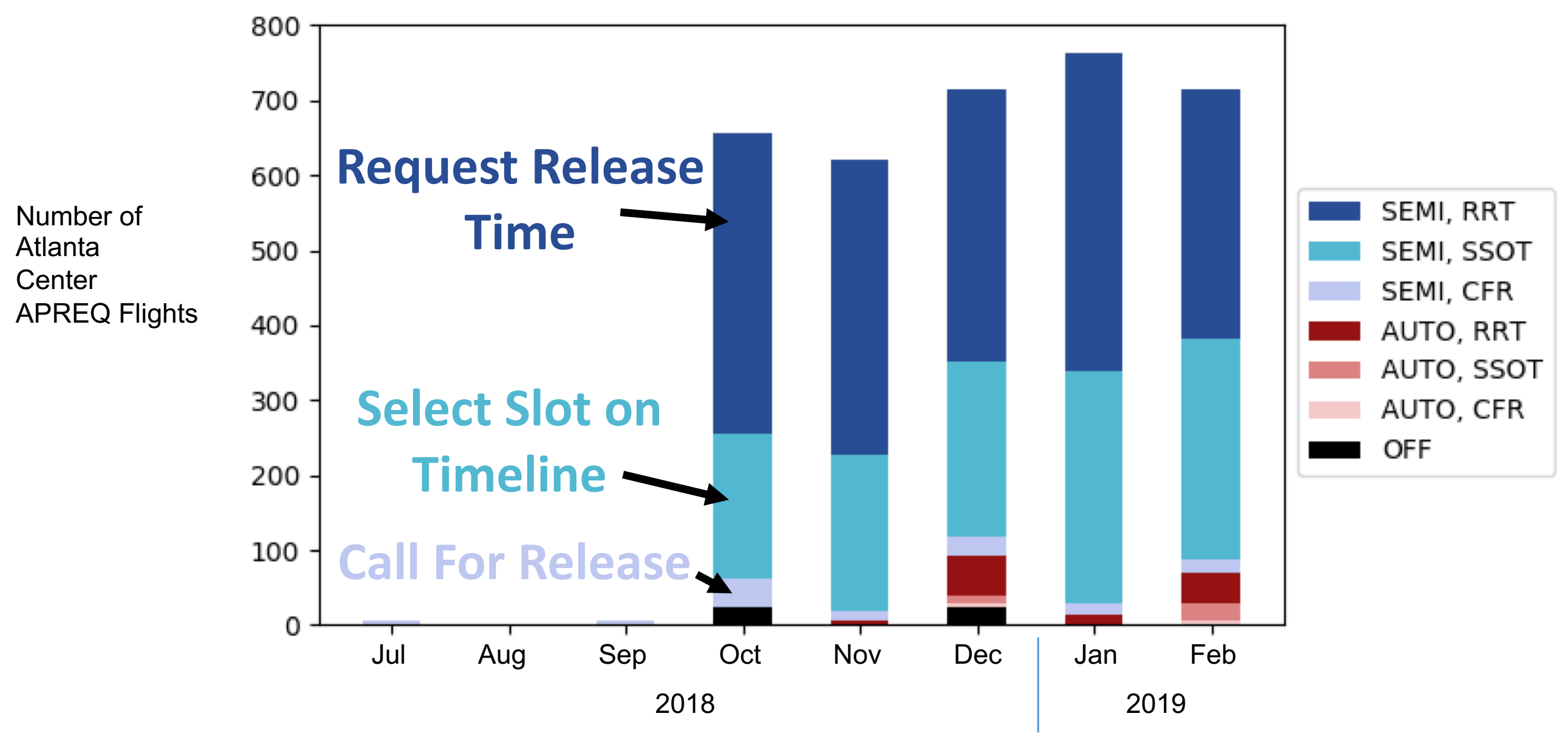




\section{Release Requests: Atlanta Center}

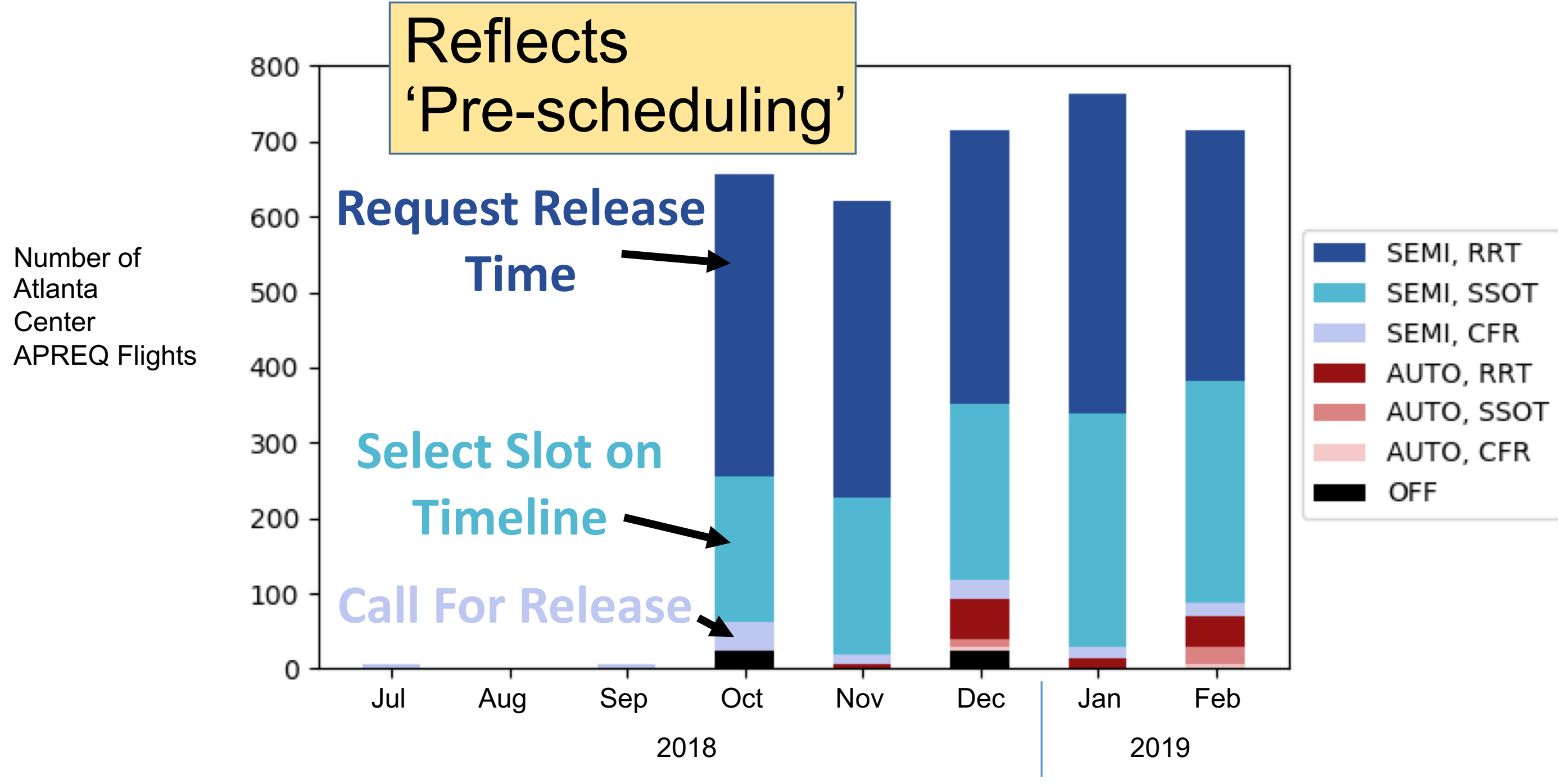




\section{APREQ Rescheduling}

- New release times negotiated for $25.9 \%$ of APREQ flights

- Of these, $30.4 \%$ received an earlier release time

Delay Savings: 73.8 hours 


\section{APREQ Aircraft Locations}

Locations at Initial Approval

Number
of Flights $\quad$ (Non-prescheduled Flights)

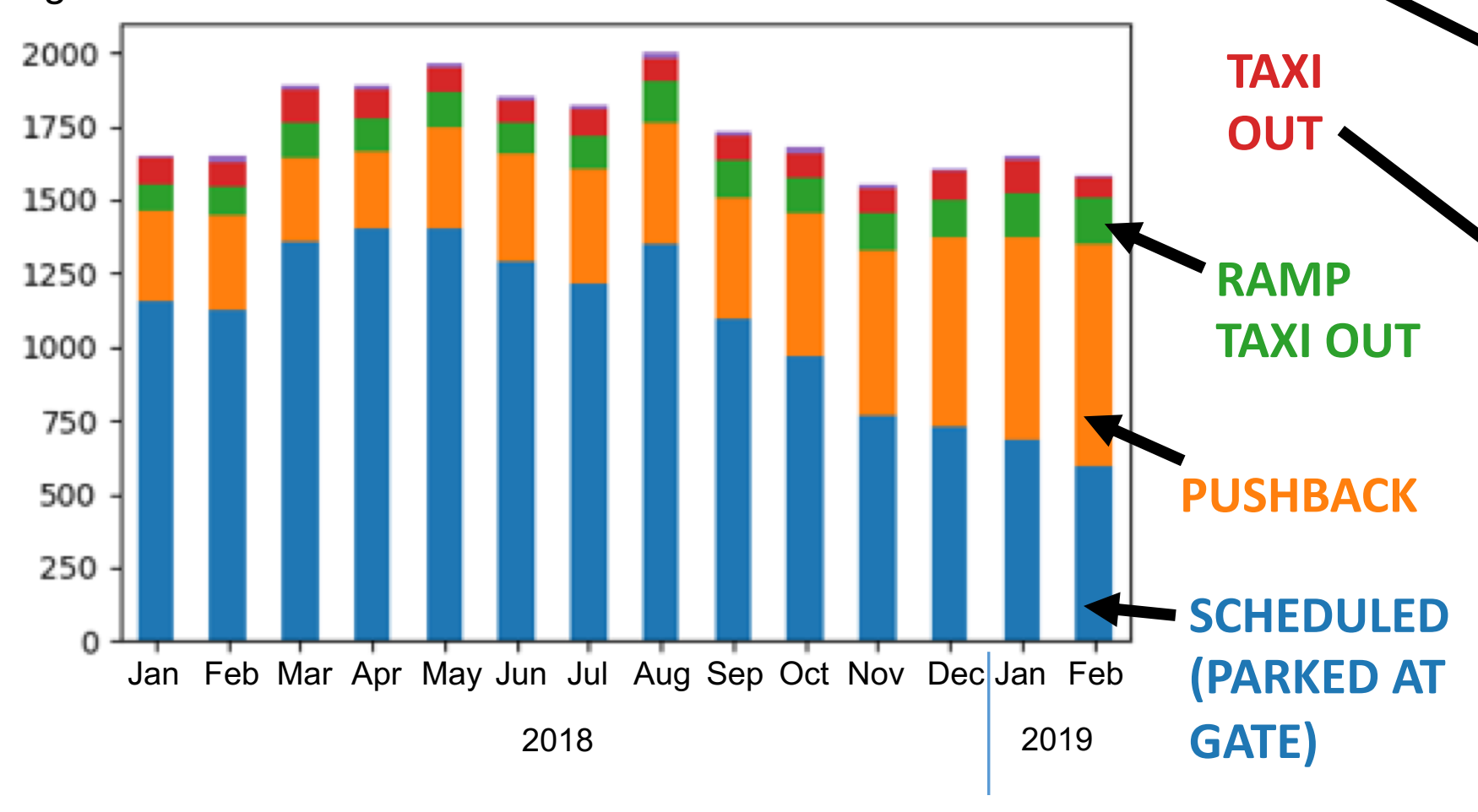

Locations at Final Approval (Rescheduled Flights)

Number
of Flights

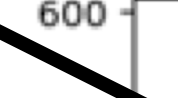

$500-$

$\underbrace{400}_{300}$

200

100

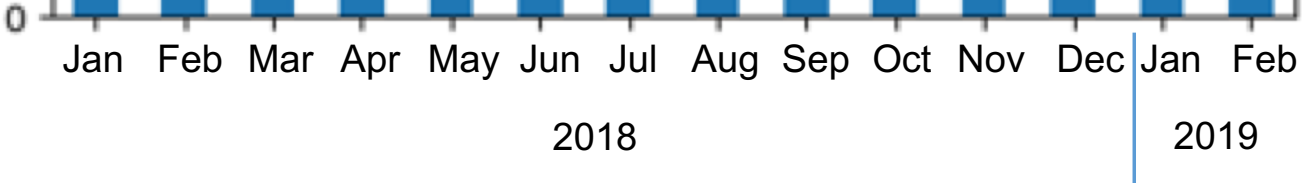




\section{Assigned Delay}

APREQ Delay $=$ Final Approved Release Time - Ltime

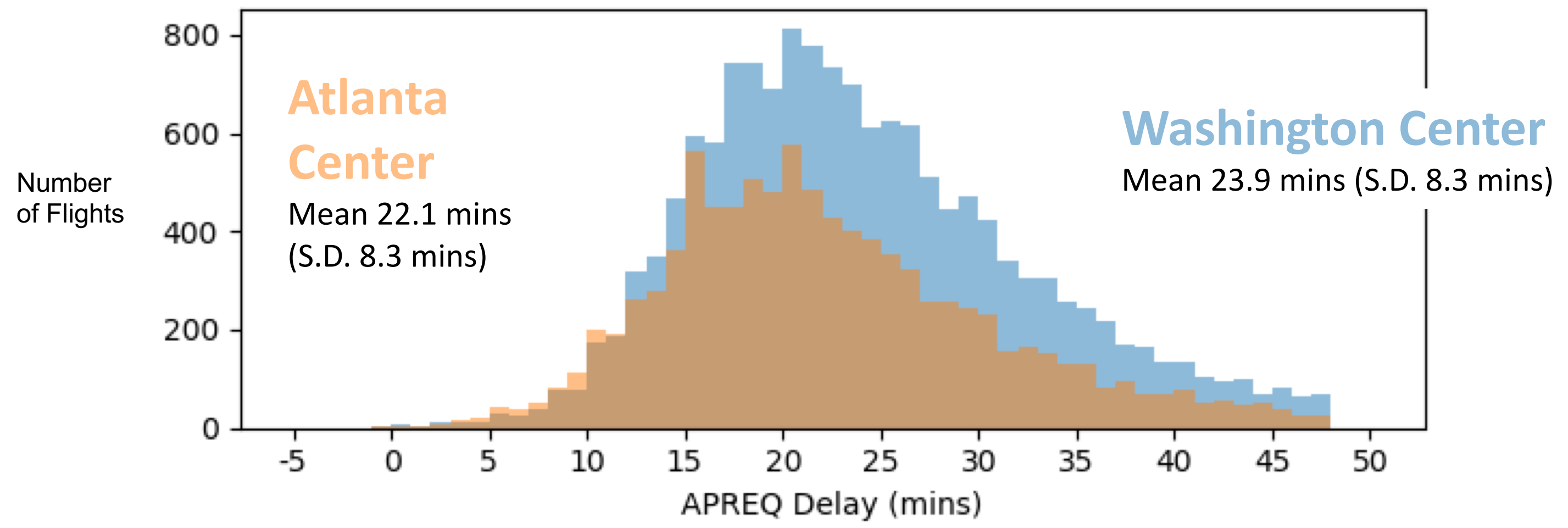

APREQ Flights with valid Ltimes; outliers removed: $90.8 \%$ of APREQ flights 


\section{APREQ Compliance}

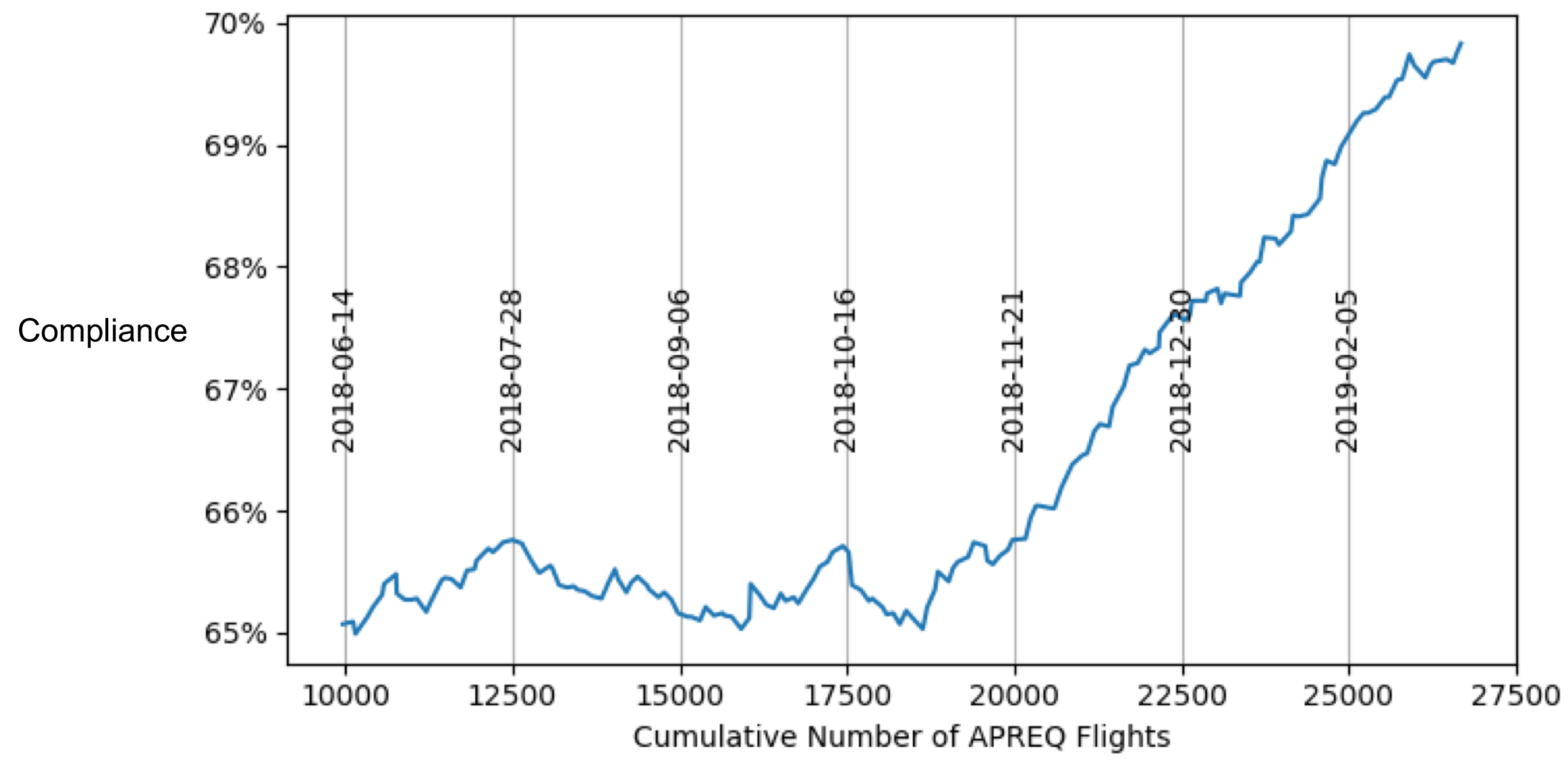




\section{APREQ Negotiation Response Times}

\section{Washington Center}

Approval Response

Time (secs)
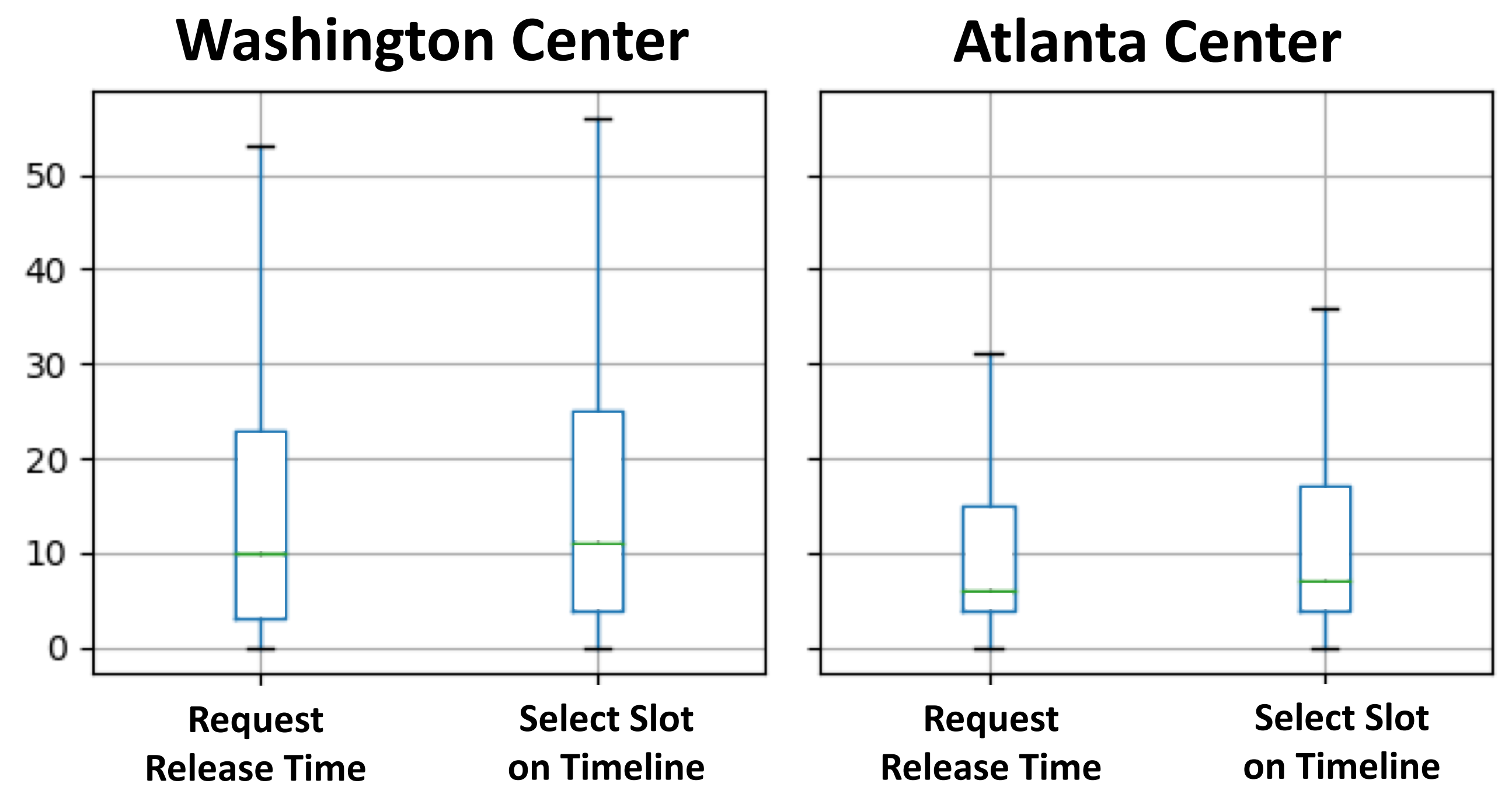


\section{Conclusions}

- $8.8 \%$ of Charlotte departures are APREQ flights

- ATD-2 STBO Client supports full range of APREQ management functions

- Most APREQs now negotiated electronically

- Fast response times

- Rescheduling flexibility

- Information sharing

- Compliance is improving

- Results support broader future deployment of similar capabilities 